\title{
Appearance of Universal Metallic Dispersion in a Doped Mott Insulator
}

\author{
S. Sahrakorpi ${ }^{1}$, R.S. Markiewicz ${ }^{1}$, Hsin $\operatorname{Lin}^{1}$, M. Lindroos ${ }^{1,2}$, X. J. Zhou ${ }^{3,4}$, T. Yoshida ${ }^{5}$, W. \\ L. Yang ${ }^{3,4}$, T. Kakeshita ${ }^{6}$, H. Eisaki ${ }^{3,6}$, S. Uchida ${ }^{6}$, Seiki Komiya ${ }^{7}$, Yoichi Ando ${ }^{8}$, F. Zhou ${ }^{9}$, \\ Z. X. Zhao ${ }^{9}$, T. Sasagawa ${ }^{3,10}$, A. Fujimori ${ }^{5}$, Z. Hussain ${ }^{4}$, Z.-X. Shen ${ }^{3,4}$, and A. Bansil ${ }^{1}$ \\ ${ }^{1}$ Physics Department, Northeastern University, Boston, Massachusetts 02115 \\ ${ }^{2}$ Institute of Physics, Tampere University of Technology, P.O. Box 692, 33101 Tampere, Finland \\ ${ }^{3}$ Dept. of Physics, Applied Physics and Stanford Synchrotron Radiation Laboratory, Stanford University, Stanford, CA 94305 \\ ${ }^{4}$ Advanced Light Source, Lawrence Berkeley National Lab, Berkeley, CA 94720 \\ ${ }^{5}$ Dept. of Physics, University of Tokyo, Bunkyo-ku, Tokyo 113-0033, Japan \\ ${ }^{6}$ Dept. of Superconductivity, University of Tokyo, Bunkyo-ku, Tokyo 113, Japan \\ ${ }^{7}$ Central Res. Inst. of Electric Power Ind., 2-11-1 Iwato-kita, Komae, Tokyo 201-8511, Japan \\ ${ }^{8}$ Institute of Scientific and Industrial Research, Osaka University, Ibaraki, Osaka 567-0047, Japan \\ ${ }^{9}$ National Lab for Superconductivity, Inst. of Physics, \\ Chinese Acad. of Sci., Beijing 100080, China and \\ ${ }^{10}$ MSL, Tokyo Institute of Technology, Kanagawa 226-8503, Japan
}

(Dated: November 12, 2018)

\begin{abstract}
We have investigated the dispersion renormalization $Z_{\text {disp }}$ in $\mathrm{La}_{2-x} \mathrm{Sr}_{x} \mathrm{CuO}_{4}$ (LSCO) over the wide doping range of $x=0.03-0.30$, for binding energies extending to several hundred meV's. Strong correlation effects conspire in such a way that the system exhibits an LDA-like dispersion which essentially 'undresses' $\left(Z_{\text {disp }} \rightarrow 1\right)$ as the Mott insulator is approached. Our finding that the Mott insulator contains 'nascent' or 'preformed' metallic states with a vanishing spectral weight offers a challenge to existing theoretical scenarios for cuprates.
\end{abstract}

PACS numbers: 79.60.-i, 71.18.+y, 74.72.Dn

Under strong electronic correlations the parent compounds of all cuprate high-temperature superconductors assume the so-called Mott-Hubbard insulating state, rather than the conventional metallic state. By what routes these insulators accomplish the miraculous transformation into superconductors with the addition of electrons or holes is a question of intense current interest, which bears on ongoing debates surrounding the interplay between electron correlations, magnetism, lattice effects, and the mechanism of high-temperature superconductivity. ${ }^{1}$ In this study we consider the classic superconductor $\mathrm{La}_{2-x} \mathrm{Sr}_{x} \mathrm{CuO}_{4}$ (LSCO) over the wide doping range of $x=0.03-0.30$, delineating how the electronic spectrum evolves with doping for binding energies extending to several hundred meV's. Our analysis indicates that this Mott insulator contains 'nascent' or 'preformed' metallic states, which develop finite spectral weight with doping, but otherwise undergo relatively little change in dispersion over a wide doping range. Our findings challenge existing theoretical scenarios for cuprates.

We have carried out extensive angle-resolved photoemission (ARPES) measurements from LSCO single crystals covering a wide range of dopings, momenta and binding energies. Although the incoherent part of the spectrum behaves quite anomalously, we find that many-body effects conspire in such a way that insofar as the coherent part of the spectrum is concerned, at least its underlying dispersion is reasonably described by the conventional band-theory picture, significantly broadened lineshapes and 'kinks' in the dispersion notwithstanding. Surprisingly, even with the addition of just a few percent holes in the insulator, the full-blown metallic spectrum seemingly turns on with little renormalization of the dispersion. In particular, the spectrum displays the presence of the telltale van Hove singularity (VHS) whose location in energy and three-dimensionality are in accord with the band theory predictions. Furthermore, this metallic spectrum is 'universal' in the sense that it depends weakly on doping, in sharp contrast to the common expectation that dispersion is renormalized to zero at half-filling.

The band structure results are based on all electron, full-potential computations within local density approximation (LDA) using the tetragonal lattice structure ${ }^{2}$, and include effects of $\mathrm{La} / \mathrm{Sr}$ substitution within the framework of the virtual crystal approximation ${ }^{3}$. The ARPES measurements were carried out on Beamline 10.0.1 at the ALS using Scienta 200, 2002, and R4000 electron energy analysers for $55 \mathrm{eV}$ light with strong inplane polarization. The energy resolution is $15-20 \mathrm{meV}$, and the angular resolution is 0.3 degrees for the 14 degrees angular mode. All data were taken at $\mathrm{T}=20 \mathrm{~K}$.

We set the stage for our discussion with the help of Fig. 1, which shows typical spectra from LSCO in the form of energy distribution curves (EDCs) at two different dopings for a series of momenta. Considering the overdoped case (upper light green set), we see a coherent feature dispersing to higher binding energies and becoming broader as one moves away from the Fermi momentum $k_{F}$. This feature sits on top of a substantial incoherent background extending to quite high energies at all momenta. These basic characteristics are seen to persist in the lightly doped sample (lower dark blue set), although the greatly reduced spectral weight of the co- 


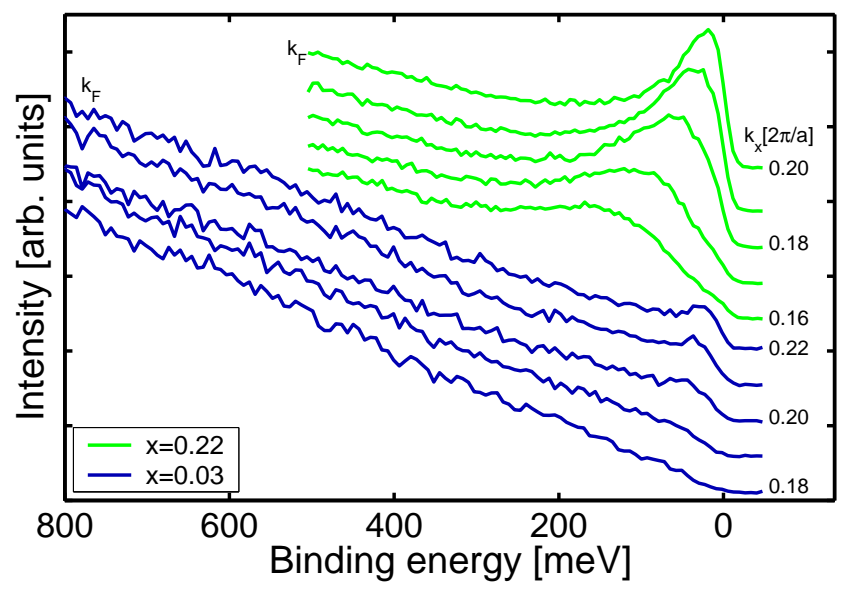

FIG. 1: (Color online) Illustrative ARPES spectra as a function of binding energy in $\mathrm{La}_{2-x} \mathrm{Sr}_{x} \mathrm{CuO}_{4}$ for a series of momenta along the nodal (i.e. $\Gamma$ to $(\pi, \pi))$ direction. Results from a lightly doped insulating sample $(x=0.03$, dark blue lines) and an overdoped metallic sample $(x=0.22$, light green lines) are shown. Coherent spectral peak is seen to disperse to higher binding energies as one moves away from the Fermi momentum, $k_{F}$.

herent feature in relation to the incoherent part of the spectrum is very evident. Our focus in this article is on the aforementioned coherent feature in the spectrum of LSCO, and especially on delineating the evolution of its dispersion with doping.

The existence of a large, Luttinger-like, metallic 'nascent' or 'underlying' Fermi surface in LSCO has been established in previous studies, culminating in the recent systematic analysis of Sahrakorpi et al. ${ }^{4}$ and Yoshida et $a l^{5}$. In contrast, here we consider spectra over a wide energy range of several hundred meV's, show the presence of the VHS-a unique feature of the band structure-even in the lightly doped insulator, and establish unequivocally the existence of near-universal metallic dispersion in LSCO. These 'nascent' Fermi surfaces and dispersions are well defined despite the difficulties of identifying features in the face of loss of spectral weight as the pseudogap develops with underdoping. We emphasize that our focus is on what we may call the 'gross' spectrum. In other words, we are not concerned with the fine structure in the electronic spectrum associated with the wellknown low energy kinks ${ }^{6}$, the recently discovered features at higher energy scales, ${ }^{7-11}$ or superconducting ${ }^{12}$, or other ${ }^{13}$ leading-edge gaps.

The top row of Fig. 2 shows ARPES intensity maps from a lightly doped sample of LSCO $(x=0.03)$ for a series of binding energies. Cross-sections of the corresponding constant energy $(\mathrm{CE})$ surfaces in the $\left(k_{x}, k_{y}\right)$ plane computed from the band structure of LSCO, superposed at $k_{z}=0$ (magenta lines) and $k_{z}=2 \pi / c$ (black lines) indicate the expected broadening of the ARPES spectra associated with interlayer coupling. At zero binding energy, i.e. the Fermi energy $E_{F}$, such $\mathrm{CE}$ contours give the projection of the 3D FS of LSCO on to the $\left(k_{x}, k_{y}\right)$ plane. Notably, the momentum region enclosed by these CE contours defines the region of allowed ARPES transitions, modulated by the effect of the ARPES matrix element. ${ }^{4,14,15}$ At low binding energies, the CE surface is seen from panels (a-c) to be hole-like around the $X(\pi, \pi)$ point for all $k_{z}$ values. In contrast, at high energy in panel (e), after the VHS has been crossed, the CE surface becomes completely electron-like centered at $\Gamma$. The transition from a hole- to electron-like $\mathrm{CE}$ surface does not take place abruptly because the VHS possess a significant 3D character, extending from $85-140 \mathrm{meV}$ in binding energy.

The evolution of the experimental ARPES intensity pattern with binding energy in the top row of Fig. 2 clearly follows that of the projected CE surfaces. In particular, the spectral intensity remains confined mainly within the boundaries of these projections as expected, and with increasing binding energy, the intensity first spreads towards the $M(\pi, 0)$-points and then moves away from the $M$-points along a perpendicular direction, very much the way the $\mathrm{CE}$ surfaces transition from being holeto electron-like. Moreover, first principles ARPES computations show that under the combined effects of the matrix element and $k_{z}$ dispersion, the spectral intensity develops the characteristic 'wing-like' shape seen in Fig. 2, and that the spectral weight grows rapidly in the antinodal region as the VHS is approached. ${ }^{4}$ These results leave no doubt that metallic states, including the presence of the 3D VHS, appear in the spectrum of the insulator with the addition of only a few percent holes, even though spectral broadening and incoherent background make it hard to see this directly in the individual EDCs. The observed location in energy and threedimensionality of the VHS is well-described by the conventional band theory picture, indicating that the energies of these metallic states undergo little renormalization in the lightly doped insulator. The evolution of the VHS over a denser binding energy mesh can be seen further in the three movies for $x=0.03,0.07$, and 0.12 provided as supplementary material. ${ }^{16}$ The characteristic dispersion of the VHS is quite recognizable in the emission maps of the top row of Fig. 2. and in the supplementary movies.

We demonstrate next that the aforementioned metallic dispersion is only weakly dependent on doping. Note that if this is true then the main difference in going from one doping to another would be a shift in the Fermi energy needed to accommodate the right number of holes in the filled portion of the band structure. That is, topologies of the $\mathrm{CE}$ surfaces and the associated emission spectra would be comparable for various dopings except for a rigid shift of the energy scales. That this is indeed the case is shown by the results for emission from the $E_{F}$ for $x=0.05-0.30$, presented in the bottom row of Fig. 2 . For example, based on parameter free LDA computations for $x=0.03$ and $x=0.30$, the $E_{F}$ for $x=0.30$ is lower by $190 \mathrm{meV}$ than for $x=0.03$, so that the ARPES map for emission at a binding energy of $190 \mathrm{meV}$ from $x=0.03$ in 


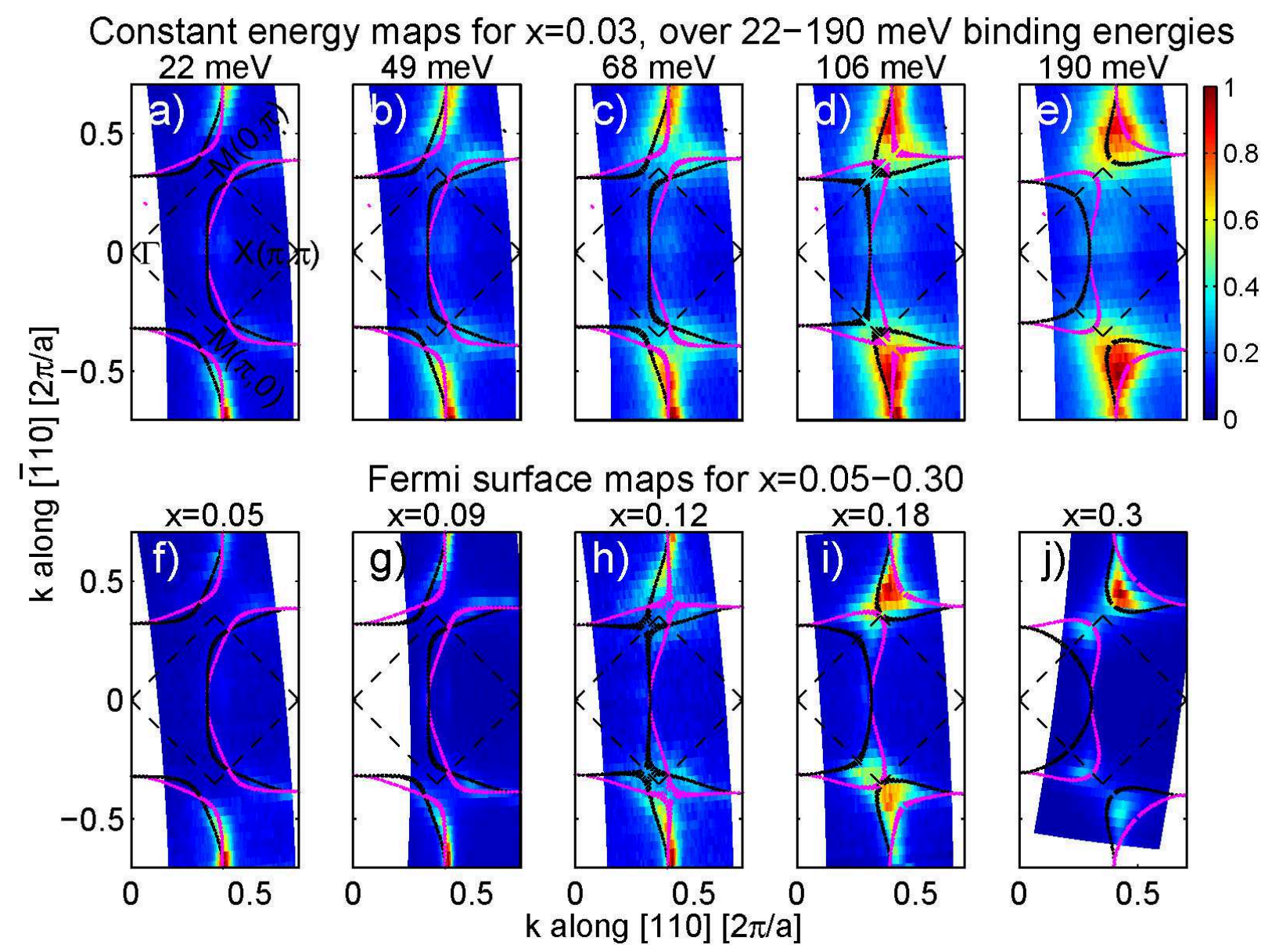

FIG. 2: (Color) Experimental ARPES intensity maps in LSCO are compared with the corresponding cuts in the $\left(k_{x}, k_{y}\right)$ plane through the theoretical constant energy (CE) surfaces for $k_{z}=0$ (magenta lines), and $k_{z}=2 \pi / c$ (black lines). Top Row: $x=0.03$ with binding energy varying from $22 \mathrm{meV}$ to $190 \mathrm{meV}$. Bottom Row: ARPES maps for emission from the Fermi energy for dopings varying from $x=0.05$ to $x=0.30$.

panel (e) can be compared with that for emission from the $E_{F}$ for $x=0.30$ in $(\mathrm{j})$. In this vein, the binding energies in the various panels of the top row of Fig. 2 for $x=0.03$ have been chosen to match the $E_{F}$ shifts involved at the doping levels considered in the panels of the bottom row. Good accord is seen in all cases. We emphasize that the energy shifts corresponding to the respective panels of the top and bottom rows in Fig. 2 are based on parameter free first principles computations. These and other similar comparisons among spectra taken at different binding energies and doping levels show clearly that LSCO is characterized by a near-universal metallic dispersion despite dramatic changes in the lineshape due to interactions. This is also true for the theoretical dispersions, although slight doping dependencies can be seen for example by comparing the $\mathrm{CE}$ surfaces in the top and bottom frames (c) and (h) in Fig. 2.

Further insight is provided by Fig. 3, which shows plots of spectral intensity as a function of binding energy along the antinodal line (top row) and the nodal line (bottom row) for six different dopings. The corresponding energy bands at $k_{z}=0, \pi / c$ and $2 \pi / c$ are overlaid in each panel. These bands are seen to differ substantially for different $k_{z}$ values along the antinodal line in the upper panels but are virtually indistinguishable in the lower panels along the nodal line. The VHS, which is marked by the extremum of the band along the antinodal line, is spread over $40 \mathrm{meV}$ at $x=0.03$ doping due to the effect of $k_{z}$ dispersion, and its center lies at $110 \mathrm{meV}$ below $E_{F}$ for $x=0.03$, but moves to $110 \mathrm{meV}$ above the $E_{F}$ for $x=0.30$ with a spread of $70 \mathrm{meV}$. Along the nodal direction, the theoretical band follows the experimental peak positions given by the black dots reasonably well, although the experimental points are shifted to the right compared to theory in most cases, indicating a slight deviation of FS shape from LDA. Along the antinodal direction, however, the spectral peaks are substantially broader due to $k_{z}$ dispersion and also possible many body interactions, although most of the spectral weight lies within the $\mathrm{k}_{z}$ dispersed bands as expected in 


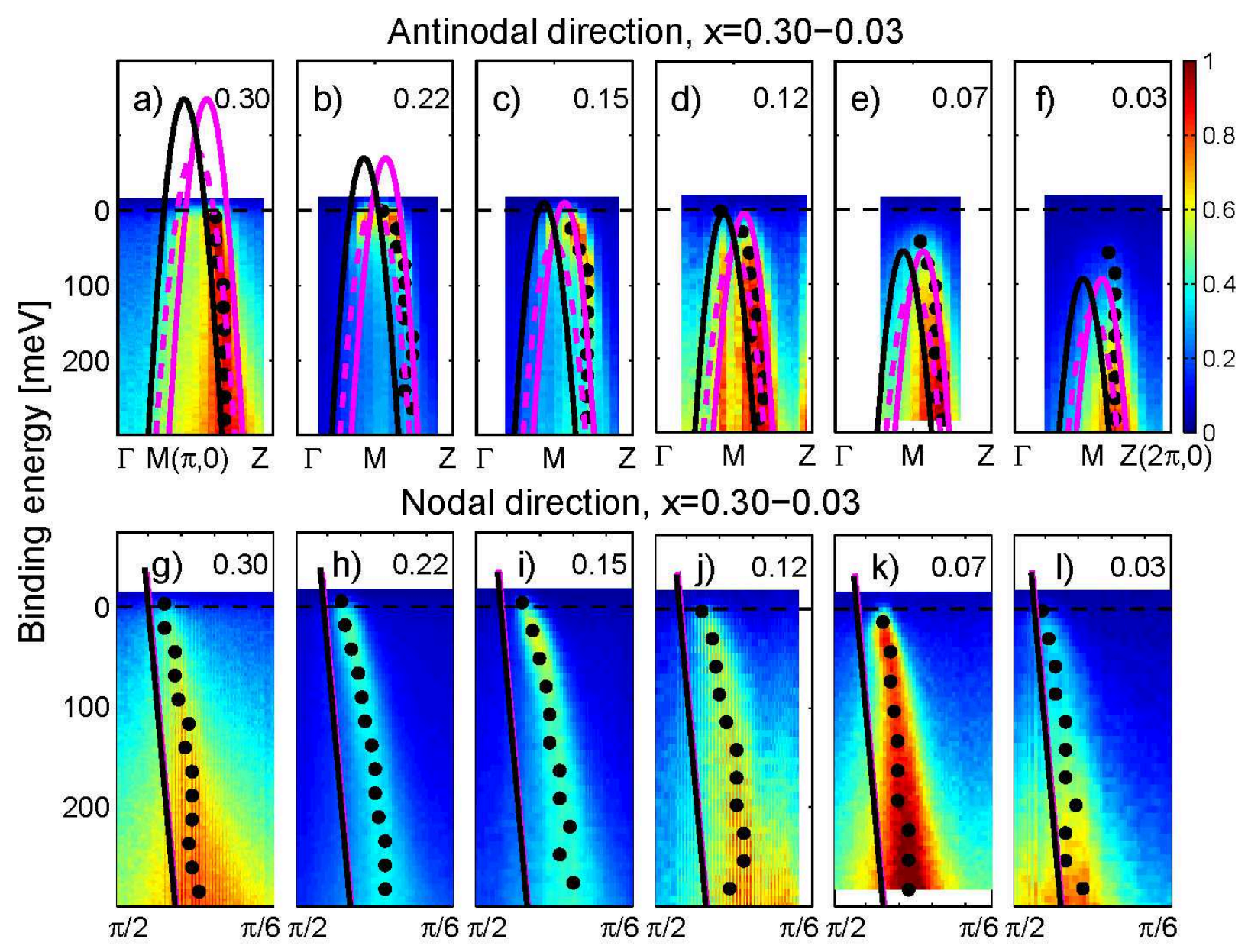

FIG. 3: (Color) ARPES intensity maps along the antinodal (top row) and nodal (bottom row) directions over the doping range $x=0.30-0.03$. The corresponding computed band structures are also plotted for three different values of $k_{z}: k_{z}=0$ (magenta solid line), $k_{z}=\pi / c$ (magenta dashed line), and $k_{z}=2 \pi / c$ (black solid line). Black dots mark positions of the peaks in the experimental spectra. Note that nodal data in the lower row are plotted on an expanded horizontal scale in order to highlight relatively small differences between gross theoretical and experimental dispersions. For each doping, the nodal and antinodal intensities are normalized to a common maximum.

\begin{tabular}{|l|c|c|c|c|c|c|}
\hline Doping $x=$ & 0.03 & 0.07 & 0.12 & 0.15 & 0.22 & 0.30 \\
\hline$Z_{\text {disp }}$ (nodal) & 1.0 & 0.8 & 0.6 & 0.6 & 0.6 & 0.6 \\
& $(1.2)$ & $(1.2)$ & $(0.8)$ & $(0.8)$ & $(0.7)$ & $(0.6)$ \\
\hline$Z_{\text {disp }}$ (antinodal) & $1.1 \pm 0.2$ & $1.3 \pm 0.4$ & $>0.6^{*}$ & $>0.6^{*}$ & $>0.7^{*}$ & $>0.7^{*}$ \\
\hline
\end{tabular}

TABLE I: Estimates of dispersion renormalization factors $Z_{\text {disp }}$ in LSCO for different dopings $x$ in relation to the LDA values. Nodal $Z_{\text {disp }}$ values in parenthesis are an estimate of the upper limit. Stars in the second row denote that for these dopings the values refer to the region in the vicinity of the antinodal point as discussed in the text.

a quasi-2D system. ${ }^{4,14,15}$

We have examined the renormalization of dispersion in relation to the LDA values along the nodal and antinodal directions as a function of doping, and thus obtained the

associated renormalization factors $Z_{\text {disp }}$. These results are summarized in Table I. Our study provides new insight into the value of $Z_{\text {disp }}$ along the antinodal direction because the VHS is a very robust feature of the LDA band structure. By determining the position of the VHS in the experimental spectrum, and comparing this position with that expected from the LDA, we can uniquely determine the overall renormalization of the spectrum along the antinodal direction with respect to the LDA. At $x=0.03$ and $x=0.07$, the VHS lies below the Fermi energy for all $k_{z}$ values, and in these two cases, by analyzing the supplementary movies ${ }^{16}$ using different renormalization factors to scale the LDA bands, we have obtained $Z_{\text {disp }}$ (antinodal) values of $\sim 1$ within the uncertainty shown in Table I, so that the LDA bands are essentially unrenormalized. For $x=0.12$ and higher dopings, part or all of the VHS lies above the Fermi energy, so that we were only able to estimate a lower limit for $Z_{\text {disp }}$ in the 


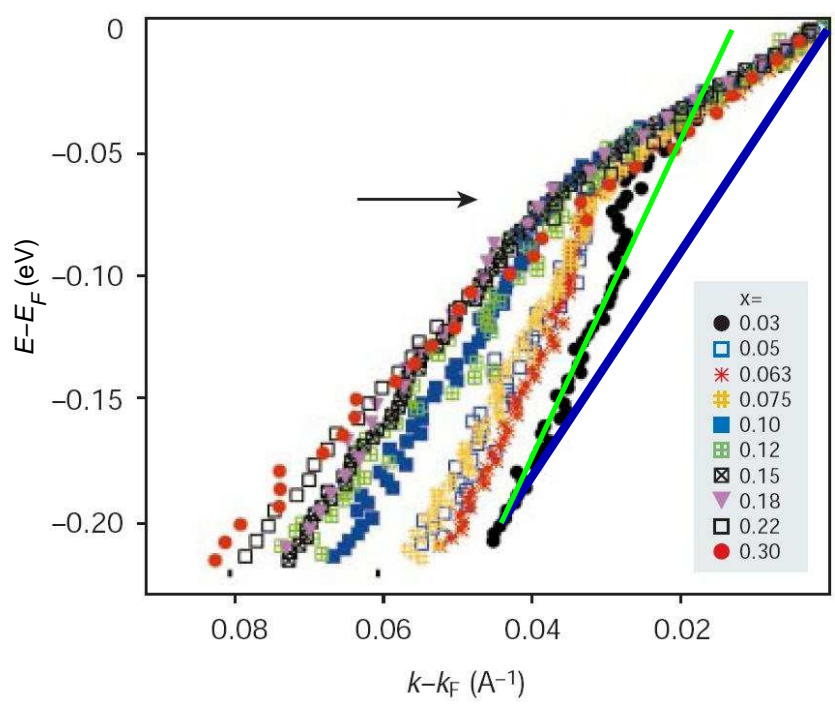

FIG. 4: (Color online) Reproduction of Fig. 1 in Zhou et al. ${ }^{17}$ Dark blue line drawn on the $x=0.03$ dataset illustrates how the nodal $Z_{\text {disp }}$ value is defined here, while the light green line gives the high energy slope used to estimate the corresponding upper limit on $Z_{\text {disp }}$ (nodal) as discussed in the text.

vicinity of the antinodal point. There are of course no filled states at the antinodal point once the VHS moves above the Fermi energy.

Turning to the nodal direction, we note that here the effects of $k_{z}$ dispersion are small, and our analysis is consistent with results available in the literature. For completeness, however, we have estimated the gross $Z_{\text {disp }}$ (nodal) values from the nodal spectra given in Fig. 1 of Zhou et al. ${ }^{17}$, reproduced here as Fig. 4. In order to gain a handle on the 'gross' or underlying dispersion exclusive of the low energy kink (see arrow at $70 \mathrm{meV}$ in Fig. 4), values of $Z_{\text {disp }}$ (nodal) given in Table I are obtained from the slopes of the straight lines joining the point at the Fermi energy with that at $200 \mathrm{meV}$ binding energy, i.e. by lines such as the dark blue line drawn for the $x=0.03$ case in Fig. 4 , and comparing this slope to the corresponding LDA values. Since the LDA values of the nodal radii do not exactly match the measured values, we have estimated $Z_{\text {disp }}$ (nodal) by comparing LDA and experimental slopes. We also show in parentheses the values of $Z_{\text {disp }}$ (nodal) obtained from the slopes of the high energy part of the spectrum, as given for example by the light green line for $x=0.03$ in Fig. 4, which provides an estimate for the upper limit of $Z_{\text {disp }}$ (nodal).

Interestingly, at the lowest doping of $x=0.03$, the nodal as well as the antinodal renormalization factor is seen to be $\sim 1$. In the optimally and overdoped regimes, the nodal renormalization factor is $\sim 0.6$, while the value of the antinodal factor is estimated to be greater than 0.6. In the underdoped $x=0.03$ case, the renormalization of states in the antinodal and nodal directions is roughly similar, but this is less clear at higher dopings. These results are surprising since we might have expected the LDA to provide a reasonable description in the overdoped regime, and to be renormalized greatly in the underdoped case.

Even though we have shown that the gross dispersion up to several hundred meV's is described quite well by the band theory picture, we emphasize that this does not mean that the spectrum of LSCO is conventional in nature. As shown in Fig. 1, the coherent spectral weight of these dispersive features fades away for underdoping where the pseudogap and polaronic effects kick in. The spectral weight is transferred to an incoherent feature ${ }^{18-20}$, reflecting the importance of many-body physics. Our analysis thus suggests that the spectrum of the insulator already contains 'preformed' or 'nascent' metallic states, which possess zero spectral weight in the half-filled case. With doping, these states develop finite spectral weight, but otherwise undergo relatively little change in their basic character.

It is interesting to study the relationship between the renormalization of the dispersion and spectral weight within the simple Green function formalism. The Green function can be approximately written as $G=Z_{\omega} /[\omega-$ $\left.Z_{\omega} \epsilon_{k} / Z_{k}\right]$, with $Z_{\omega}=1 /(1-\partial \Sigma / \partial \omega)$, and $Z_{k}=1 /(1+$ $\left.\partial \Sigma / \partial \epsilon_{k}\right)$, where $\Sigma$ is the self-energy. While the spectral weight renormalization is associated with $Z_{\omega}$, the band dispersion renormalization is given by $Z_{d i s p}=Z_{\omega} / Z_{k}$. It is striking that the spectral weight in LSCO is renormalized greatly but the dispersion is not. This is not consistent with a momentum independent self-energy, since the renormalization of spectral weight would then be accompanied by a similar renormalization of dispersion, suggesting that the self-energy possesses momentum as well as energy dependencies such that the effects of the associated renormalization factors, $Z_{\omega}$ and $Z_{k},{ }^{21}$ conspire to approximately cancel each other in renormalizing dispersion. This picture however implies that the heat capacity would be renormalized only weakly with doping, which is not the case experimentally. ${ }^{22}$

Insofar as theoretical models of strongly correlated systems are concerned, a focus of attention in the literature has been the $t-J$ model, which has been used to describe the physics of the Hubbard Hamiltonian in the large $U$ regime. One class of $t-J$ models finds gapped insulating solutions at half-filling, which evolve with doping to yield small Fermi surface pockets ${ }^{23}$. This route appears to be followed in $\mathrm{Nd}_{2-x} \mathrm{Ce}_{x} \mathrm{CuO}$ for electron doping ${ }^{24-26}$. But the present results do not show clear evidence for small pockets or other strong violations of Luttinger's theorem ${ }^{27}$. On the other hand, metallic solutions with a large Luttinger-like Fermi surface have also been reported ${ }^{12,28}$, where the spectral weight $Z_{\omega}$ is renormalized to zero at half-filling. These solutions are closer to our observations, even though the band width is found to be strongly renormalized near half-filling, $Z_{\text {disp }} \sim J / t$. A recent variant of the $t-J$ model succeeds in capturing both the metallic and insulating aspects of the spectrum simultaneously ${ }^{29,30}$. However, to our knowledge, 
our finding of a large Fermi surface and a large dispersion, which is weakly dependent on doping, does not fit well within the framework of the currently available scenarios based on simple Hubbard or $t-J$ models or naive application of the classical polaron theory.

In conclusion, we have shown that metallic states appear in the electronic spectrum of high temperature superconductor $\mathrm{La}_{2-x} \mathrm{Sr}_{x} \mathrm{CuO}_{4}$ at all doping levels, ranging from the lightly doped insulating to the overdoped metallic regime. The gross dispersion of these states is not only near-universal in that it is weakly dependent on doping, but it is also conventional in that it is reasonably described by the standard band theory picture up to binding energies of several hundred meV's. However, the metallic states suffer substantial loss of spectral weight with underdoping and in this respect behave quite unconventionally. Our findings challenge existing theoretical models of the cuprates and indicate the complexity with which many-body physics plays out in this fascinating material.

Acknowledgments This work was supported by the US Department of Energy (DOE), Office of Basic Energy Sciences, with contracts DE-FG02-07ER46352 and DE-AC03-76SF00098, and benefited from the allocation of supercomputer time at NERSC and Northeastern University's Advanced Scientific Computation Center (ASCC). The Stanford work was supported by DOE Office of Science, Division of Materials Science, with contract DE-FG03-01ER45929-A001. A.F. and S.U. acknowledge a Grant-in-Aid for Scientific Research in Priority Area "Invention of Anomalous Quantum Materials" from MEXT, Japan for financial support. Y.A. was supported by KAKENHI 16340112 and 19674002 .
1 A. Damascelli, Z. Hussain, and Z. X. Shen, Reviews of Modern Physics 75, 473 (2003).

2 J. D. Jorgensen, H. B. Schuttler, D. G. Hinks, D. W. Capone, K. Zhang, M. B. Brodsky, and D. J. Scalapino, Physical Review Letters 58, 1024 (1987).

3 H. Lin, S. Sahrakorpi, R. S. Markiewicz, and A. Bansil, Physical Review Letters 96, 097001 (2006).

4 S. Sahrakorpi, M. Lindroos, R. S. Markiewicz, and A. Bansil, Physical Review Letters 95, 157601 (2005).

${ }^{5}$ T. Yoshida, X. J. Zhou, K. Tanaka, W. L. Yang, Z. Hussain, Z. X. Shen, A. Fujimori, S. Sahrakorpi, M. Lindroos, R. S. Markiewicz, A. Bansil, S. Komiya, Y. Ando, H. Eisaki, T. Kakeshita, and S. Uchida, Physical Review B 74, 224510 (2006).

6 A. Lanzara, P. V. Bogdanov, X. J. Zhou, S. A. Kellar, D. L. Feng, E. D. Lu, T. Yoshida, H. Eisaki, A. Fujimori, K. Kishio, J. I. Shimoyama, T. Noda, S. Uchida, Z. Hussain, and Z. X. Shen, Nature 412, 510 (2001).

7 F. Ronning, K. M. Shen, N. P. Armitage, A. Damascelli, D. H. Lu, Z. X. Shen, L. L. Miller, and C. Kim, Physical Review B 71, 094518 (2005).

8 J. Graf, G. H. Gweon, K. McElroy, S. Y. Zhou, C. Jozwiak, E. Rotenberg, A. Bill, T. Sasagawa, H. Eisaki, S. Uchida, H. Takagi, D. H. Lee, and A. Lanzara, Physical Review Letters 98, 067004 (2007).

9 W. Meevasana, X. J. Zhou, S. Sahrakorpi, W. S. Lee, W. L. Yang, K. Tanaka, N. Mannella, T. Yoshida, D. H. Lu, Y. L. Chen, R. H. He, H. Lin, S. Komiya, Y. Ando, F. Zhou, W. X. Ti, J. W. Xiong, Z. X. Zhao, T. Sasagawa, T. Kakeshita, K. Fujita, S. Uchida, H. Eisaki, A. Fujimori, Z. Hussain, R. S. Markiewicz, A. Bansil, N. Nagaosa, J. Zaanen, T. P. Devereaux, and Z. X. Shen, Physical Review B 75, 174506 (2007).

10 B. P. Xie, K. Yang, D. W. Shen, J. F. Zhao, H. W. Ou, J. Wei, S. Y. Gu, M. Arita, S. Qiao, H. Namatame, M. Taniguchi, N. Kaneko, H. Eisaki, K. D. Tsuei, C. M. Cheng, I. Vobornik, J. Fujii, G. Rossi, Z. Q. Yang, and D. L. Feng, Physical Review Letters 98, 147001 (2007).

11 T. Valla, T. E. Kidd, W. G. Yin, G. D. Gu, P. D. Johnson, Z. H. Pan, and A. V. Fedorov, Physical Review Letters 98, 167003 (2007).
12 R. Sensarma, M. Randeria, and N. Trivedi, Physical Review Letters 98, 027004 (2007).

13 A. Kanigel, M. R. Norman, M. Randeria, U. Chatterjee, S. Souma, A. Kaminski, H. M. Fretwell, S. Rosenkranz, M. Shi, T. Sato, T. Takahashi, Z. Z. Li, H. Raffy, K. Kadowaki, D. Hinks, L. Ozyuzer, and J. C. Campuzano, Nature Physics 2, 447 (2006).

14 A. Bansil and M. Lindroos, Physical Review Letters 83, 5154 (1999).

15 A. Bansil, M. Lindroos, S. Sahrakorpi, and R. S. Markiewicz, Physical Review B 71, 012503 (2005).

16 See EPAPS Document No. E-PRBMDO-78-025834 for movies showing ARPES intensity maps at a series of energies passing through the VHS, at three dopings $\mathrm{x}=0.03,0.07$, and 0.12 . These movies can be directly accessed at ftp://ftp.aip.org/epaps/phys_ rev_b/E-PRBMD0-78-025834/. For more information on EPAPS, see http://www. aip.org/pubservs/epaps.html.

17 X. J. Zhou, T. Yoshida, A. Lanzara, P. V. Bogdanov, S. A. Kellar, K. M. Shen, W. L. Yang, F. Ronning, T. Sasagawa, T. Kakeshita, T. Noda, H. Eisaki, S. Uchida, C. T. Lin, F. Zhou, J. W. Xiong, W. X. Ti, Z. X. Zhao, A. Fujimori, Z. Hussain, and Z. X. Shen, Nature 423, 398 (2003).

18 T. Yoshida, X. J. Zhou, T. Sasagawa, W. L. Yang, P. V. Bogdanov, A. Lanzara, Z. Hussain, T. Mizokawa, A. Fujimori, H. Eisaki, Z. X. Shen, T. Kakeshita, and S. Uchida, Physical Review Letters 91, 027001 (2003).

19 K. M. Shen, F. Ronning, D. H. Lu, W. S. Lee, N. J. C. Ingle, W. Meevasana, F. Baumberger, A. Damascelli, N. P. Armitage, L. L. Miller, Y. Kohsaka, M. Azuma, M. Takano, H. Takagi, and Z. X. Shen, Physical Review Letters 93, 267002 (2004).

${ }^{20}$ O. Rosch, O. Gunnarsson, X. J. Zhou, T. Yoshida, T. Sasagawa, A. Fujimori, Z. Hussain, Z. X. Shen, and S. Uchida, Physical Review Letters 95, 227002 (2005).

21 A. Paramekanti, M. Randeria, and N. Trivedi, Physical Review B 70, 054504 (2004).

${ }^{22}$ T. Matsuzaki, N. Momono, M. Oda, and M. Ido, Journal of the Physical Society of Japan 73, 2232 (2004).

23 P. Prelovsek and A. Ramsak, Physical Review B 65, 174529 (2002). 
${ }^{24}$ N. P. Armitage, D. H. Lu, C. Kim, A. Damascelli, K. M. Shen, F. Ronning, D. L. Feng, P. Bogdanov, Z. X. Shen, Y. Onose, Y. Taguchi, Y. Tokura, P. K. Mang, N. Kaneko, and M. Greven, Physical Review Letters 87, 147003 (2001).

25 C. Kusko, R. S. Markiewicz, M. Lindroos, and A. Bansil, Physical Review B 66, 140513(R) (2002).

26 A. M. S. Tremblay, B. Kyung, and D. Senechal, Low Temperature Physics 32, 424 (2006).
27 J. Kokalj and P. Prelovsek, Physical Review B 75, 045111 (2007).

28 B. Edegger, V. N. Muthukumar, C. Gros, and P. W. Anderson, Physical Review Letters 96, 207002 (2006).

29 T. C. Ribeiro and X. G. Wen, Physical Review Letters 95, 057001 (2005).

${ }^{30}$ F. Tan, Y. Wan, and Q. H. Wang, Physical Review B 76, 054505 (2007). 